\title{
25 Research Soure \\ Combination of Serological Biomarkers and Clinical Features To Predict Mucosal Healing in Crohn's Disease: A Multicenter Cohort Study
}

Nana Tang

Jiangsu Province Hospital and first affiliated hospital of Nanjing Medical University https://orcid.org/0000-0002-4688-3043

Han Chen

Jiangsu Province Hospital and Nanjing Medical University First Affiliated Hospital

Ruidong Chen

Second Affiliated Hospital of Soochow University

Wen Tang

Second Affiliated Hospital of Soochow University

Hongjie Zhang ( $\nabla$ hjzhang06@163.com )

Jiangsu Province Hospital and first affiliated hospital of Nanjing Medical University

Research

Keywords: Crohn's disease, mucosal healing, nomogram, PLR, endoscopic

Posted Date: November 2nd, 2021

DOI: https://doi.org/10.21203/rs.3.rs-985096/v1

License: (c) (i) This work is licensed under a Creative Commons Attribution 4.0 International License.

Read Full License 


\section{Abstract \\ Background}

Mucosal healing (MH) has become the treatment goal of patients with Crohn's disease (CD). This study aims to develop a noninvasive and reliable clinical tool for individual evaluation of mucosal healing in patients with Crohn's disease.

\section{Results}

The following variables were independently associated with the $\mathrm{MH}$ and were subsequently included into the prediction model: PLR (platelet to lymphocyte ratio), CAR (C-reactive protein to albumin ratio), ESR (erythrocyte sedimentation rate), HBI (Harvey-Bradshaw Index) score and infliximab treatment. A primary model and a simple model were established, respectively. The primary model performed better than the simple one in C-index ( $87.5 \%$ vs $83.0 \%, p=0.004)$. There was no statistical significance between these two models in sensitivity $(70.43 \%$ vs $62.61 \%, p=0.467)$, specificity $(87.12 \%$ vs $80.69 \%, p=0.448)$, PPV (72.97\% vs $61.54 \%, p=0.292)$, NPV ( $85.65 \%$ vs $81.39 \%, p=0.614)$, and accuracy $(81.61 \%$ vs $74.71 \%$, $\mathrm{p}=0.303)$. The primary model had good calibration and high levels of explained variation and discrimination in validation cohort.

\section{Conclusions}

This model can be used to predict $\mathrm{MH}$ in post-treatment $\mathrm{CD}$ patients. It can also be used as an indication of endoscopic surveillance to evaluate mucosal healing in patients with CD after treatment.

\section{Background}

Crohn's disease (CD) is a life-long and progressive inflammatory disease with symptoms evolving in a remitting and relapsing manner ${ }^{1}$. Most patients develop bowel damage and disability including strictures, fistulas, abscesses and so on in several years after diagnosis, resulting in surgery. ${ }^{2}$ Current therapeutic strategies in CD aim for deep and prolonged remission, with the goal of preventing complications and therefore improve prognosis as well as quality of life. ${ }^{3}$ Treatment strategies aimed solely at resolution of clinical symptoms does not eliminate long-term bowel damage in CD patients. ${ }^{4}$

Mucosal healing $(\mathrm{MH})$ is confirmed lead to a lower rate of relapse, hospitalization and surgical resection. ${ }^{5}$ In recent years, $\mathrm{MH}$ is preferred over clinical remission as straightforward goal of clinical treatment in CD. ${ }^{6}$ Endoscopy is still the golden standard for evaluation of disease activity. However, frequently endoscopy for disease monitoring in long-term follow-up is limited by considerations of invasiveness, high cost and patient acceptance. Alternative noninvasive methods are necessary for assessment of CDrelated mucosal inflammation. ${ }^{7,8}$ 
It is reported that clinical characteristics such as mild clinical manifestation and early introduction of biologicals associated with $\mathrm{MH}$ in patients with $\mathrm{CD} .{ }^{9-11}$ In addition, some systemic inflammatory markers obtained from the serological examination including neutrophil to lymphocyte ratio (NLR) and platelet to lymphocyte ratio $(\mathrm{PLR})^{12}$, C-reactive protein to Albumin ratio $(\mathrm{CAR})^{13}$, combination of fecal calprotectin $(F C)$, erythrocyte sedimentation rate (ESR), C-reactive protein (CRP) and Albumin (ALB) ${ }^{14}$ have been explored as diagnostic and predictive indicators of $C D$. Clinically, there is still lack of accurate instrument with high sensitivity and specificity using serological parameters and clinical characteristics to predict $\mathrm{MH}$ in $\mathrm{CD}$.

In this study, we aimed to address the predictive role of serum inflammatory index and clinical features in CD patients who diagnosed and treated in two tertiary hospitals in China. We analyzed the pre-treatment and post-treatment data individually and explored their relationship with $\mathrm{MH}$ after treatment in patients with CD. Subsequently, we used hematological data with or without clinical features to construct assessment models for $\mathrm{MH}$ prediction. Model with superior performance is recommended for clinical use.

\section{Results}

\section{Demographic and Clinical Characteristics of Patients}

A total of 348 patients with CD were enrolled into present study and 115 patients achieved $\mathrm{MH}$. Baseline demographic and clinical characteristics are shown in Table 1. The median diagnosis age of the included patients was 28.0 years (IQR:21-39years) and mean disease course of all the individuals was 26.3 months. Median HBI score of the patients was 7 (IQR:5-9). Shapiro-Wilk test showed that data of diagnosis age, disease course and $\mathrm{HBI}$ score were with abnormal distributions (all $p<0.001$ ). Thus, MannWhitney $\mathrm{U}$ test was performed and identified that levels of $\mathrm{HBI}(p=0.006)$ and age at diagnosis $(p=0.003)$ were significantly associate with $\mathrm{MH}$, while the disease course was not associated with $\mathrm{MH}(p=0.893)$. In addition, chi-square analyze showed that patients without lumen stenosis $(p=0.005)$ and treatment with infliximab $(p<0.001)$ are associated with $\mathrm{MH}$.

TABLE 1. Demographic and Clinical Characteristics of MH and non-MH Patients 
Mucosal healing Non-Mucosal healing P-value

$\begin{array}{llll} & \mathrm{N}(\%) & \mathrm{N}(\%) & \\ \text { Number of patients } & 115(33) & 233(67) & 0.120 \\ \text { Gender } & & & \\ \text { Male } & 85(35.7) & 153(64.3) & 80(72.7)\end{array}$

Smoking

0.701

\begin{tabular}{llll}
\hline Non-smoker & $99(32.7)$ & $204(67.3)$ & \\
Smoker & $16(35.6)$ & $29(64.4)$ & 1.000 \\
\hline $\begin{array}{l}\text { Family history of IBD } \\
\text { No }\end{array}$ & $113(33.1)$ & $228(66.9)$ & \\
Yes & $2(28.6)$ & $5(71.4)$ & 0.799 \\
\hline Surgical history & & & \\
No & $95(32.8)$ & $195(67.2)$ & 0.055 \\
Yes & $20(34.5)$ & $38(65.5)$ & \\
\hline Disease location & & & \\
\hline L1 lleal & & $71(63.4)$ & \\
\hline L2 Colonic & $41(36.6)$ & $111(64.5)$ & \\
\hline L3 Ileocolonic & $13(20.3)$ & & \\
\hline
\end{tabular}

Upper digestive tract involved

0.412

No

92(32.4)

192(67.6)

Yes

24(37.5)

$40(62.5)$

Stenosis

0.005

No

97(37.2)

164(62.8)

Yes

18(20.7)

69(79.3)

Penetrating 


\begin{tabular}{|c|c|c|c|}
\hline No & $225(68.0)$ & $106(32.0)$ & \\
\hline Yes & $8(47.1)$ & $9(52.9)$ & \\
\hline Perianal lesion & & & 0.626 \\
\hline No & $59(31.9)$ & $126(68.1)$ & \\
\hline Yes & $56(34.4)$ & $107(65.6)$ & \\
\hline
\end{tabular}

Medication treatment

Corticosteroids

0.953

No

98(33.1)

198(66.9)

Yes

17(32.7)

35(67.3)

Immunomodulators

0.72

No

95(32.6)

196(67.4)

Yes

20(35.1)

37(64.9)

Infliximab

$[0.001$

No

39(17.4)

185(82.6)

Yes

76(61.3)

48(38.7)

\section{Pre-treatment hematological parameters and Mucosal Healing}

Pre-treatment laboratory blood parameters of CD patients were summarized in Table 2. Shapiro-Wilk test showed that all the data of blood test were with abnormal distributions (all $p<0.001)$. Thus Mann-Whitney $\mathrm{U}$ test was performed and identified that the levels of Eosinophils $(p=0.021), \operatorname{MLR}(p=0.020), \operatorname{PLR}$ $(p=0.015)$ and CAR $(p=0.044)$ were significantly associate with $\mathrm{MH}$. Furthermore, these significant factors were selected to further perform multivariate regression analysis and only PLR could evaluate $\mathrm{MH}$ after treatment $(p=0.037)$. The ROC curve analysis showed that AUC of PLR were 0.58 (95\% Cl: 0.515-0.644, $P=0.015)$. The sensitivity was 0.815 and specificity was 0.313 .

TABLE 2. Logistic regression for hematological parameters evaluation of $\mathrm{MH}$ 


\begin{tabular}{lllllll}
\multicolumn{3}{l}{ Pre-treatment } & \multicolumn{3}{l}{ Post-treatment } \\
\hline Blood & Non-MH & MH & P-value & Non-MH & MH & P- \\
tests & M (Q) & M (Q) & & $M(Q)$ & $M(Q)$ & value \\
\hline WBC & $7.02(3.43)$ & $7.23(3.60)$ & 0.276 & $6.27(2.70)$ & $5.86(2.23)$ & 0.205 \\
\hline NE & $5.19(2.82)$ & $5.44(2.74)$ & 0.985 & $4.17(2.18)$ & $3.39(1.75)$ & $\square 0.001$ \\
\hline MO & $0.54(0.35)$ & $0.52(0.28)$ & 0.790 & $0.97(0.26)$ & $0.42(0.20)$ & 0.001 \\
\hline EO & $0.20(0.12)$ & $0.26(0.17)$ & 0.021 & $0.21(0.13)$ & $0.12(0.08)$ & 0.209 \\
\hline BA & $0.03(0.02)$ & $0.03(0.02)$ & 0.687 & $0.04(0.03)$ & $0.02(0.02)$ & 0.239 \\
\hline HGB & $117.9(34.8)$ & $121.8(31.0)$ & 0.161 & $125.1(34)$ & $134.1(23)$ & $\square 0.001$ \\
HCT & $42.29(9.33)$ & $37.29(9.1)$ & 0.582 & $38.5(8.45)$ & $40.4(6.7)$ & 0.003 \\
PLT & $304.9(128)$ & $300.57(155)$ & 0.943 & $275.9(120)$ & $230.3(79)$ & $\square 0.001$ \\
\hline CRP & $25.87(31.7)$ & $24.65(30.76)$ & 0.086 & $17.2(17.33)$ & $3.41(2.15)$ & $\square 0.001$ \\
ESR & $29.52(34.0)$ & $27.09(35.0)$ & 0.139 & $25.9(30.4)$ & $9.54(11.0)$ & $\square 0.001$ \\
NLR & $4.41(2.45)$ & $6.52(2.56)$ & 0.112 & $3.69(2.29)$ & $2.03(1.07)$ & $\square 0.001$ \\
MLR & $0.42(0.30)$ & $0.53(0.24)$ & $\mathbf{0 . 0 2 0}$ & $0.67(0.25)$ & $0.25(0.15)$ & $\square 0.001$ \\
PLR & $242(154.2)$ & $475.8(148)$ & $\mathbf{0 . 0 1 5}$ & $228.6(124)$ & $137.1(77.4)$ & $\square 0.001$ \\
CAR & $0.80(0.98)$ & $0.72(0.82)$ & $\mathbf{0 . 0 4 4}$ & $0.49(0.48)$ & $0.09(0.06)$ & $\square 0.001$ \\
\hline PAR & $8.89(5.24)$ & $8.20(5.39)$ & 0.209 & $7.46(3.44)$ & $5.52(2.11)$ & $\square 0.001$
\end{tabular}

Abbreviations: $\mathrm{MH}$, mucosal healing; WBC, White Blood Cell; NE, Neutrophils; MO, Monocyte; EO, Eosinophils; BA, Basophils; HGB, Hemoglobin; HCT, hematocrit; PLT, platelet; CRP, C reactive protein; ESR, erythrocyte sedimentation rate; NLR, Neutrophil-Lymphocyte Ratio; MLR, Monocyte-Lymphocyte Ratio; PLR, Platelet-Lymphocyte Ratio; CAR, C reactive protein-Albumin Ratio; PAR, Platelet- Albumin Ratio;

\section{Post-treatment hematological parameters and Mucosal Healing}

Shapiro-Wilk test showed that all the data of blood test after treatment were with abnormal distributions (all $p<0.001)$. Mann-Whitney $\mathrm{U}$ test identified that the levels of $\mathrm{NE}(p<0.001), \mathrm{MO}(p=0.001)$, $\operatorname{HGB}(p<0.001), \operatorname{HCT}(p=0.003), \operatorname{PLT}(p<0.001), \operatorname{CRP}(p<0.001), \operatorname{ESR}(p<0.001), \operatorname{NLR}(p<0.001), \operatorname{MLR}(p<0.001)$, $\operatorname{PLR}(p<0.001), \operatorname{CAR}(p<0.001), \operatorname{PAR}(p<0.001)$ were significantly associate with $\mathrm{MH}(\mathrm{Table} 2)$. In the multivariate regression, we identified the following three variables as the independently associated factors with $M H$ : PLR, CAR and ESR.

\section{Model establishment}


We established two models: a simple model and a primary model. The simple model (model-1) only contains serum biomarkers including PLR, CAR and ESR. The primary model (model-2) was consisted of the following variables: PLR, CAR, ESR, HBI score and treatment with infliximab. Variables included in the simple and primary models are showed in Table3.

TABLE 3. Multivariate logistic regression of models for Mucosal healing evaluation

\begin{tabular}{lllll} 
& \multicolumn{2}{l}{ Simple Model (model-1) } & \multicolumn{2}{l}{ Primary Model (model-2) } \\
\hline OR [95\%Cl] & $p$-Value & OR [95\%Cl] & $p$-Value \\
\hline HGB & $0.996[0.971-1.021]$ & 0.754 & $0.986[0.952-1.022]$ & 0.437 \\
\hline HCT & $0.968[0.877-1.068]$ & 0.519 & $0.978[0.861-1.112]$ & 0.734 \\
NE & $0.909[0.754-1.096]$ & 0.317 & $0.847[0.676-1.060]$ & 0.147 \\
\hline MO & $0.950[0.756-1.194]$ & 0.661 & $0.848[0.120-5.984]$ & 0.848 \\
CAR & $0.022[0.002-0.219]$ & $\mathbf{0 . 0 0 1}$ & $0.036[0.004-0.320]$ & 0.003 \\
PLR & $0.993[0.989-0.997]$ & $\mathbf{0 . 0 0 1}$ & $0.995[0.990-0.999]$ & 0.014 \\
\hline ESR & $0.955[0.928-0.982]$ & $\mathbf{0 . 0 0 1}$ & $0.951[0.922-0.981]$ & $\mathbf{0 . 0 0 2}$ \\
Age & NA & NA & $0.993[0.967-1.021]$ & 0.682 \\
HBI & NA & NA & $0.907[0.824-0.999]$ & $\mathbf{0 . 0 4 7}$ \\
Stenosis & NA & NA & $0.599[0.289-1.241]$ & 0.168 \\
\hline Infliximab & NA & NA & $6.346[3.324-12.117]$ & 00.001
\end{tabular}

\section{Comparisons between simple model and primary model}

Diagnostic value was compared between the two models. The golden standard is whether $\mathrm{MH}$ has been achieved under endoscopy. Suppl.Table1 shows the classification of the two models.

The C-index of simple model was 0.830 (95\% Cl: 0.79-0.87, P<0.001) (Fig.1A). The sensitivity and specificity were 0.626 and 0.807 , respectively (Table.4). Primary model showed a perfect capacity for predicting MH, with a C-index of 0.875 (95\% Cl: 0.84-0.91, P<0.001) (Fig.1A). Sensitivity of primary model was 0.704 and specificity was 0.871 (Table.4). According to DeLong's test, there is significant difference of $\mathrm{C}$-index between primary model and simple model $(\mathrm{Z}=2.8519, \mathrm{P}=0.0043)$. Primary model was superior to simple model in C-index $(87.5 \%$ vs $83.0 \%, p=0.004)$. Although there was no statistical significance, primary model has an advantage over simple model with a higher sensitivity $(70.43 \%$ vs $62.61 \%, p=0.467)$, specificity ( $87.12 \%$ vs $80.69 \%, p=0.448)$, PPV ( $72.97 \%$ vs $61.54 \%, p=0.292)$, NPV $(85.65 \%$ vs $81.39 \%, p=0.614)$, and accuracy $(81.61 \%$ vs $74.71 \%, p=0.303)$ (Table 4$)$. 
TABLE 4. Comparison of simple model and primary model

\begin{tabular}{|llll|}
\hline Diagnostic Index & $\begin{array}{l}\text { Simple Model } \\
(\%, 95 \% \mathrm{Cl})\end{array}$ & $\begin{array}{l}\text { Primary Model } \\
(\%, 95 \% \mathrm{Cl})\end{array}$ & $\begin{array}{l}\text { P-value } \\
\text { C-index }\end{array}$ \\
& 0.830 & 0.875 & $0.004^{\star}$ \\
& $(0.79-0.87)$ & $(0.84-0.91)$ & \\
\hline Sensitivity & $62.61 \%$ & $70.43 \%$ & 0.467 \\
& $(53.10-71.45 \%)$ & $(61.21-78.58 \%)$ & \\
Specificity & $80.69 \%$ & $87.12 \%$ & 0.448 \\
& $(75.02-85.55 \%)$ & $(82.13-91.14 \%)$ & \\
\hline PPV & $61.54 \%$ & $72.97 \%$ & 0.292 \\
& $(54.29-68.31 \%)$ & $(65.45-79.37 \%)$ & \\
\hline NPV & $81.39 \%$ & $85.65 \%$ & 0.614 \\
& $(77.39-84.81 \%)$ & $(81.76-88.83 \%)$ & \\
\hline Accuracy & $74.71 \%$ & $81.61 \%$ & 0.303 \\
& $(69.80-79.20 \%)$ & $(77.13-85.54 \%)$ & \\
\hline
\end{tabular}

Note: *Statistically significant with a p-value less than 0.05 .

Abbreviations: Simple Model: model constructed from PLR, CAR and ESR; Primary Model: model constructed from PLR, CAR, ESR, HBI and IFX treatment; PPV, positive predictive value; NPV, negative predictive value.

\section{Evaluation and Validation of the Primary Nomogram}

A nomogram was established based on the variables in the primary model (Fig.2). Model performance was evaluated by discrimination and calibration. This model had a high C-index (0.88) as mentioned above. The calibration curve also showed satisfactory performance (figure $3 \mathrm{~A}$ ). The internal validation was performed in $112 \mathrm{CD}$ patients with small bowel involvement. After validation, the $\mathrm{C}$-index of the model was 0.834 (95\% Cl: $0.76-0.91, P<0.001)$ (Fig.1B). The calibration curve in validation group is shown in Fig.3B. The internal validation also performed good in discrimination and calibration.

\section{Discussion}

In the present study, the most important findings include: (1) Serum biomarkers such as PLR, CAR and ESR after approximately 1 year treatment was independently associated with $\mathrm{MH}$. (2) Patients with lower 
$\mathrm{HBI}$ score and the use of infliximab are more likely to achieve $\mathrm{MH}$. Based on this, we developed prediction model with the above significant variables to evaluate $\mathrm{MH}$ in $\mathrm{CD}$ patients.

The routine blood test is the most fundamental and accessible examination, which has long been proposed as an essential assistant tool for disease assessment. ${ }^{15}$ In our study, there was no statistical significance between pre-treatment serum inflammatory indexes and $\mathrm{MH}$. Thus, we were unable to develop a pre-treatment model. However, in the post-treatment data, we found the combination use of PLR, CAR and ESR can effectively evaluate MH in CD patients. Platelet count can be affected by cytokines released in acute inflammation. Thrombocytosis and high ESR level are common feature of acute inflammation. Lymphocytes is the basic component of the adaptive and innate immune system. It is demonstrated that PLR increased significantly in endoscopically active ulcerative colitis. ${ }^{16} \mathrm{CRP}$ is the most widely used serological indicators in clinical evaluation of disease activity in CD. ${ }^{17,18}$ Serum ALB is an indicator of nutrition, synthesis rate of which directly affected by the severity of acute infection. CAR was initially used to identify critical patients in emergency ward and predict disease progression in Takayasu arteritis and cancer in recent years. ${ }^{19,} 20$ It is reported that CAR is useful biomarker of disease activity and histological activity in $\mathrm{CD} .{ }^{21}$ Consistent with previous research, we included the above three variables including PLR, CAR and ESR into our model.

Some clinical characteristics can also predict MH in CD patients. In is reported that early introduction of tumor necrosis factor (TNF) antagonists, particularly in combination with immunosuppressives associate with $\mathrm{MH}$ in patients with $\mathrm{CD} .{ }^{22}$ In our study, patients received infliximab was significantly associate with an increased rate of $\mathrm{MH}$, consistent with previous reports. $\mathrm{HBI}$ was derived to simplify calculation of the Crohn's disease activity index (CDAl). We newly found that $\mathrm{HBI}$ was associated with $\mathrm{MH}$. In univariate logistic regression analyses, diagnose age and lumen stenosis were found associate with $\mathrm{MH}$ in the present data. Unfortunately, neither of them was included in the final multivariate logistic regression model.

A simple model and a primary model were established in the study. The simple model is simpler, easier to operate clinically, and with favorable accuracy. However, taking into account of the C-index and the calibration plots, primary model showed better discrimination ability. Reliable nomogram based on aforementioned factors was constructed and showed excellent evaluation abilities for $\mathrm{MH}$ among $\mathrm{CD}$ patients. Parameters in the monogram are easy to obtain, which increases the clinical practicality. This nomogram can predict $\mathrm{MH}$ probability in $\mathrm{CD}$ patients after one year of treatment and provide reference for doctors to perform endoscopic review. If the prediction results indicate low probability of $\mathrm{MH}$, doctors could temporarily eliminate endoscopy and adjust treatment regimen, avoiding repeated and unnecessary invasive endoscopy.

Fecal calprotectin (FC) has been widely clarified for the correlation with endoscopically proven CD activity. ${ }^{23-25}$ However, FC is still not commonly used in clinical practice because of detection results may vary from different kits of calprotectin. In addition, some researchers pointed out that PPV of FC for $\mathrm{MH}$ was not high enough and FC was not sensitive to assess CD activity with small intestine involvement. ${ }^{26}$, 
${ }^{27}$ For these reasons, we did not include FC in present study. Nevertheless, we validated primary model in $\mathrm{CD}$ patients with small intestine involvement, indicating a good evaluation effect.

Our study has some limitations. Firstly, although the patients we included from two tertiary hospitals in Eastern China, the results may not represent the general population of CD patients. Secondly, only internal validation was performed in the present study. Results of present study still need to be verified by external large-scale clinical studies with follow-up study.

\section{Conclusion}

In summary, this study provides comprehensive insights into serum inflammatory index and clinical information to evaluate $\mathrm{MH}$ after treatment in $\mathrm{CD}$ patients. We conducted a nomogram, providing a portable decision tool for early $\mathrm{MH}$ screening and clinical decision of endoscopic review time. More prospective studies in the future are warrant to perform.

\section{Methods}

\section{The aim, design and setting of the study}

We aim to develop a noninvasive and reliable nomogram for individual prediction of mucosal healing in patients with Crohn's disease. A multicenter retrospective cohort was established, composed of 348 consecutive patients with CD between January 2010 and June 2021. Clinical and serological variables were collected. Separate risk factors were incorporated into a binary logistic regression model. The model performance was evaluated with C-index, sensitivity, specificity, positive predictive value (PPV), negative predictive value (NPV) and accuracy. Internal validation was performed in 112 patients with small intestinal lesions.

\section{Patients and Data source}

This was a retrospective, multi-center observational cohort study of consecutive CD patients from Inflammatory Bowel Disease Center of The First Affiliated Hospital of Nanjing Medical University and the Second Affiliated Hospital of Soochow University, China, between 2010 and 2021. Diagnoses of CD were determined according to standard clinical, laboratory, radiological, endoscopic, and histopathologic

findings. ${ }^{28}$ Data regarding patients' demographics, laboratory values and endoscopic characteristics were retrospectively reviewed through hospital medical database records and endoscopic image system. Harvey-Bradshaw Index ( $\mathrm{HBI}$ ) consists of five descriptors: general well-being, abdominal pain, number of liquid stools for the previous day, abdominal mass and complications. ${ }^{29}$ The HBI score was calculated at baseline of diagnosis.

Inclusion criteria were listed as follows: (1) Patients underwent at least twice endoscopic procedures and serological examination both pre-treatment and post-treatment during the study period. (2) Corticosteroids had been discontinued for more than 12 weeks. Exclusion criteria: (1) Acute or chronic 
infections during the inspection; (2) Previous medical history of hematologic or rheumatic autoimmune disease; (3) Acute or chronic renal failure, heart diseases, cirrhosis or cancer; (4) A previous history of taking aspirin or warfarin; (5) Missing complete blood count, ALB, ESR or CRP data. (6) Any other conditions that affect the blood routine results or inflammatory markers. The clinical, endoscopic features and laboratory data of the study population are summarized in Tables 1 and 2.

Ethical approval for the study was approved by Clinical Research Ethics Committee of the hospital (ref: 2021-SR-235), in compliance with the Declaration of Helsinki. All patients in the study gave their informed consent for reviewing their clinical data.

\section{Blood assessment and Endoscopic documentation}

Baseline blood values had been collected at the time of CD diagnosis when patients were admitted to hospital before administration of any treatment. Post-treatment hematology was completed within one week of the patient's endoscopic review. Venous blood specimens were drawn into sterile standard tubes containing ethylene diamine tetraacetic acid (EDTA) as an anticoagulant and evaluated within $1 \mathrm{~h}$ after venipuncture using a Beckman Coulter UniCel DxH800 hematology analyzer. The Beckman Coulter UniCel ${ }^{\circledR}$ DxH 800 was used for analyzing ESR and routine blood markers including White Blood Cell (WBC), neutrophils (NE), monocytes (MO), lymphocytes (LY), Eosinophils (EO), Basophilic (BA), Hemoglobin (HGB), platelet (PLT) and hematocrit (HCT). The Beckman Coulter AU5800 Clinical Chemistry Analyzer was used for assessing ALB and CRP. Inflammatory markers of NLR, Monocyte-to-Lymphocyte Ratio (MLR), PLR, CRP-ALB Ratio (CAR) and Platelet-ALB Ratio (PAR) were calculated subsequently.

Patients underwent at least twice endoscopic examination during the study, before treatment and approximately one year after treatment (10-14 months), respectively. Endoscopic procedures were performed with the standard protocol and the static endoscopic images were reassessed retrospectively by two experienced gastroenterologists. $\mathrm{MH}$ was defined as a mucosal activity of gastrointestinal tract as remission or mild inflammatory activity, without ulcer. ${ }^{30}$ Disease phenotype was established according to Montreal Classification. ${ }^{31,32}$

\section{Statistical Analysis}

The statistical analyses were performed by using SPSS 26.0 software (SPSS, Chicago, IL, USA). Normality test were applied by Shapiro-Wilk test. Data with normal distribution are presented as mean with Standard deviation (SD), and data with non-normal distribution are presented as median with Interquartile Range (Q). The t test (2-tailed) was applied for data with normal distribution while MannWhitney $U$ test were performed in data with abnormal distribution. Chi-square tests or Fisher's exact test were used to compare the nonparametric categorical data between groups.

Univariate and multivariant analyses were applied in SPSS. R software (version 3.3.2) was used to build the nomogram and evaluation of model performance ("rms" package). Parameters inclusive of the interaction terms and of clinical significance were included in a full multivariate model subsequently. The 
model performance was evaluated with C-index, sensitivity, specificity, positive predictive value (PPV), negative predictive value (NPV) and accuracy. To simplify the logistic regression results and create a practical tool, the coefficients derived from the multivariate analysis were used as weights to elaborate a nomogram, which facilitates the practical application of the model for evaluating probability of $\mathrm{MH}$ expected for a given patient. Internal validation was performed in patients with small intestinal lesions. $P$ value less than 0.05 was considered statistically significant.

\section{List Of Abbreviations}

MH Mucosal healing

CD Crohn's Disease

CDAl Crohn's Disease Activity Index

HBI Harvey Bradshaw Index

$\mathrm{Cl}$ confidence interval

PPV positive predictive value

NPV negative predictive value

WBC white blood cell

NE Neutrophil

MO Monocyte

EO Eosnophil

BA Basophilic granulocyte

HGB Hemoglobin

HCT Hematocrit

PLT platelet

CRP C reactive protein

ESR Erythrocyte Sedimentation Rate

ALB albumin

FC fecal calprotectin 
NLR Neutrophil/lymphocyte ratio

MLR Monocyte /lymphocyte ratio

PLR platelet-lymphocyte ratio

CAR C-reactive protein/Albumin ratio

PAR platelet/albumin ratio

\section{Declarations}

\section{Ethics approval and consent to participate}

Ethical approval for the study was approved by Clinical Research Ethics Committee of The First Affiliated Hospital of Nanjing Medical University, China(ref:2021-SR-235), in compliance with the Declaration of Helsinki. All patients in the study gave their informed consent for reviewing their clinical data. The letter of consent for ethical review is as follows:

\section{Consent for publication}

The manuscript is approved by all authors for publication. The manuscript has not been published previously, in any language, in whole or in part, and is not currently under consideration elsewhere.

\section{Availability of data and material}

The datasets during and analysed during the current study available from the corresponding author on reasonable request.

\section{Competing interests}

No conflict of interest exits in the submission of this manuscript

\section{Funding}

This study was supported by grants from the National Natural Science Foundation of China, No. 81770553 and 82070568.

\section{Authors' contributions}

Nana Tang, Han Chen and Ruidong Chen have contributed equally to this manuscript. N.T. and H.C. wrote the manuscript. N.T. and R.C. contributed to data accumulation. H.C. and N.T. performed statistical analysis. H.Z. and W.T. have participated in the study design. H.Z. critically revised the manuscript.

\section{Acknowledgements}




\section{References}

1. Hanzel, J., A Novel Endoscopic Score for Postoperative Recurrence of Crohn's Disease: More Information Needed. Am J Gastroenterol 2021, 116 (1), 217-218.

2. Ho, G. T.; Cartwright, J. A.; Thompson, E. J.; Bain, C. C.; Rossi, A. G., Resolution of Inflammation and Gut Repair in IBD: Translational Steps Towards Complete Mucosal Healing. Inflamm Bowel Dis 2020, $26(8), 1131-1143$.

3. Nunez, F. P.; Mahadevan, U.; Quera, R.; Bay, C.; Ibanez, P., Treat-to-target approach in the management of inflammatory Bowel disease. Gastroenterol Hepatol 2021, 44 (4), 312-319.

4. Lafeuille, P.; Hordonneau, C.; Vignette, J.; Blayac, L.; Dapoigny, M.; Reymond, M.; Rouquette, O.; Sollelis, E.; Boube, M.; Magnin, B.; Pereira, B.; Buisson, A., Transmural healing and MRI healing are associated with lower risk of bowel damage progression than endoscopic mucosal healing in Crohn's disease. Aliment Pharmacol Ther 2021, 53 (5), 577-586.

5. Dal Buono, A.; Roda, G.; Argollo, M.; Zacharopoulou, E.; Peyrin-Biroulet, L.; Danese, S., Treat to target or 'treat to clear' in inflammatory bowel diseases: one step further? Expert Rev Gastroenterol Hepatol 2020, 14 (9), 807-817.

6. Cucchiara, S.; D'Arcangelo, G.; Isoldi, S.; Aloi, M.; Stronati, L., Mucosal healing in Crohn's disease: new insights. Expert Rev Gastroenterol Hepatol 2020, 14 (5), 335-345.

7. Zhou, F. S.; Gao, N.; Sun, X.; Jiang, X. Y.; Chen, J. J.; Mao, Q. Q.; Zhong, L., C-reactive protein/abumin ratio is a useful biomarker for predicting the mucosal healing in the Crohn disease: A retrospective study. Medicine (Baltimore) 2021, 100 (10), e24925.

8. FGC, E. P.; Rosa, R. M.; da Cunha, P. F. S.; de Souza, S. C. S.; de Abreu Ferrari, M. L., Faecal calprotectin is the biomarker that best distinguishes remission from different degrees of endoscopic activity in Crohn's disease. BMC Gastroenterol 2020, 20 (1), 35.

9. Colombel, J. F.; Adedokun, O. J.; Gasink, C.; Gao, L. L.; Cornillie, F. J.; D'Haens, G. R.; Rutgeerts, P. J.; Reinisch, W.; Sandborn, W. J.; Hanauer, S. B., Combination Therapy With Infliximab and Azathioprine Improves Infliximab Pharmacokinetic Features and Efficacy: A Post Hoc Analysis. Clin Gastroenterol Hepatol 2019, 17 (8), 1525-1532 e1.

10. Dulai, P. S.; Boland, B. S.; Singh, S.; Chaudrey, K.; Koliani-Pace, J. L.; Kochhar, G.; Parikh, M. P.; Shmidt, E.; Hartke, J.; Chilukuri, P.; Meserve, J.; Whitehead, D.; Hirten, R.; Winters, A. C.; Katta, L. G.; Peerani, F.; Narula, N.; Sultan, K.; Swaminath, A.; Bohm, M.; Lukin, D.; Hudesman, D.; Chang, J. T.; Rivera-Nieves, J.; Jairath, V.; Zou, G. Y.; Feagan, B. G.; Shen, B.; Siegel, C. A.; Loftus, E. V., Jr.; Kane, S.; Sands, B. E.; Colombel, J. F.; Sandborn, W. J.; Lasch, K.; Cao, C., Development and Validation of a Scoring System to Predict Outcomes of Vedolizumab Treatment in Patients With Crohn's Disease. Gastroenterology 2018, $155(3), 687-695$ e10. 
11. Mao, R.; Qiu, Y.; Chen, B. L.; Zhang, S. H.; Feng, R.; He, Y.; Zeng, Z. R.; Ben-Horin, S.; Chen, M. H., Factors associated with the achievement of mucosal healing in Crohn's disease: the benefit of endoscopic monitoring in treating to target. Therap Adv Gastroentero/ 2017, 10 (6), 453-463.

12. Feng, J. R.; Qiu, X.; Wang, F.; Chen, P. F.; Gao, Q.; Peng, Y. N.; Lin, X.; Liu, Q.; Liu, J.; Zhao, Q.; Li, J., Diagnostic Value of Neutrophil-to-Lymphocyte Ratio and Platelet-to-Lymphocyte Ratio in Crohn's Disease. Gastroenterol Res Pract 2017, 2017, 3526460.

13. Chen, Y. H.; Wang, L.; Feng, S. Y.; Cai, W. M.; Chen, X. F.; Huang, Z. M., The Relationship between CReactive Protein/Albumin Ratio and Disease Activity in Patients with Inflammatory Bowel Disease. Gastroenterol Res Pract 2020, 2020, 3467419.

14. Daniluk, U.; Daniluk, J.; Krasnodebska, M.; Lotowska, J. M.; Sobaniec-Lotowska, M. E.; Lebensztejn, D. M., The combination of fecal calprotectin with ESR, CRP and albumin discriminates more accurately children with Crohn's disease. Adv Med Sci 2019, 64 (1), 9-14.

15. Sun, S.; Karsdal, M. A.; Mortensen, J. H.; Luo, Y.; Kjeldsen, J.; Krag, A.; Jensen, M. D.; Bay-Jensen, A. C.; Manon-Jensen, T., Serological Assessment of the Quality of Wound Healing Processes in Crohn's Disease. J Gastrointestin Liver Dis 2019, 28, 175-182.

16. Akpinar, M. Y.; Ozin, Y. O.; Kaplan, M.; Ates, I.; Kalkan, I. H.; Kilic, Z. M. Y.; Yuksel, M.; Kayacetin, E., Platelet-to-lymphocyte Ratio and Neutrophil-to-lymphocyte Ratio Predict Mucosal Disease Severity in Ulcerative Colitis. J Med Biochem 2018, 37(2), 155-162.

17. Lin, X.; Qiu, Y.; Feng, R.; Chen, B.; He, Y.; Zeng, Z.; Zhang, S.; Chen, M.; Mao, R., Normalization of CReactive Protein Predicts Better Outcome in Patients With Crohn's Disease With Mucosal Healing and Deep Remission. Clin Trans/ Gastroenterol 2020, 11 (2), e00135.

18. Ma, C.; Battat, R.; Khanna, R.; Parker, C. E.; Feagan, B. G.; Jairath, V., What is the role of C-reactive protein and fecal calprotectin in evaluating Crohn's disease activity? Best Pract Res Clin Gastroenterol 2019, 38-39, 101602.

19. Seringec Akkececi, N.; Yildirim Cetin, G.; Gogebakan, H.; Acipayam, C., The C-Reactive Protein/Albumin Ratio and Complete Blood Count Parameters as Indicators of Disease Activity in Patients with Takayasu Arteritis. Med Sci Monit 2019, 25, 1401-1409.

20. Wu, J.; Tan, W.; Chen, L.; Huang, Z.; Mai, S., Clinicopathologic and prognostic significance of Creactive protein/albumin ratio in patients with solid tumors: an updated systemic review and metaanalysis. Oncotarget 2018, 9 (17), 13934-13947.

21. Nassri, A.; Muftah, M.; Nassri, R.; Fialho, A.; Fialho, A.; Ribeiro, B.; Ghali, P., Novel InflammatoryNutritional Biomarkers as Predictors of Histological Activity in Crohn's Disease. Clin Lab 2020, 66 (7).

22. Vasudevan, A.; Raghunath, A.; Anthony, S.; Scanlon, C.; Sparrow, M. P.; Gibson, P. R.; van Langenberg, D. R., Higher Mucosal Healing with Tumor Necrosis Factor Inhibitors in Combination with Thiopurines Compared to Methotrexate in Crohn's Disease. Dig Dis Sci 2019, 64 (6), 1622-1631.

23. Leach, S. T.; Day, A. S.; Messenger, R.; Walters, T. D.; Navas-Lopez, V. M.; Sladek, M.; Bruckner, A.; Yerushalmi, B.; Saeed, S.; Otley, A.; Mack, D.; Gavish, M.; Turner, D.; Griffiths, A. M.; Lemberg, D. A.; 
ImageKids study, g., Fecal Markers of Inflammation and Disease Activity in Pediatric Crohn Disease: Results from the ImageKids Study. J Pediatr Gastroenterol Nutr 2020, 70 (5), 580-585.

24. Kawashima, K.; Ishihara, S.; Yuki, T.; Fukuba, N.; Sonoyama, H.; Kazumori, H.; Yamashita, N.; Tada, Y.; Kusunoki, R.; Oka, A.; Oshima, N.; Mishima, Y.; Moriyama, I.; Kinoshita, Y., Fecal Calprotectin More Accurately Predicts Endoscopic Remission of Crohn's Disease than Serological Biomarkers Evaluated Using Balloon-assisted Enteroscopy. Inflamm Bowel Dis 2017, 23 (11), 2027-2034.

25. Kennedy, N. A.; Jones, G. R.; Plevris, N.; Patenden, R.; Arnott, I. D.; Lees, C. W., Association Between Level of Fecal Calprotectin and Progression of Crohn's Disease. Clin Gastroenterol Hepato/ 2019, 17 (11), 2269-2276 e4.

26. Costa, F.; Mumolo, M. G.; Ceccarelli, L.; Bellini, M.; Romano, M. R.; Sterpi, C.; Ricchiuti, A.; Marchi, S.; Bottai, M., Calprotectin is a stronger predictive marker of relapse in ulcerative colitis than in Crohn's disease. Gut 2005, 54 (3), 364-8.

27. Verdejo, C.; Hervias, D.; Roncero, O.; Arias, A.; Bouhmidi, A.; Lorente, R.; Saluena, I.; Lucendo, A. J., Fecal calprotectin is not superior to serum C-reactive protein or the Harvey-Bradshaw index in predicting postoperative endoscopic recurrence in Crohn's disease. Eur J Gastroenterol Hepatol 2018, $30(12), 1521-1527$.

28. Alkhatry, M.; Al-Rifai, A.; Annese, V.; Georgopoulos, F.; Jazzar, A. N.; Khassouan, A. M.; Koutoubi, Z.; Nathwani, R.; Taha, M. S.; Limdi, J. K., First United Arab Emirates consensus on diagnosis and management of inflammatory bowel diseases: A 2020 Delphi consensus. World J Gastroenterol 2020, 26 (43), 6710-6769.

29. Levesque, B. G.; Sandborn, W. J.; Ruel, J.; Feagan, B. G.; Sands, B. E.; Colombel, J. F., Converging goals of treatment of inflammatory bowel disease from clinical trials and practice. Gastroenterology 2015, 148 (1), 37-51 e1.

30. af Bjorkesten, C. G.; Nieminen, U.; Turunen, U.; Arkkila, P. E.; Sipponen, T.; Farkkila, M. A., Endoscopic monitoring of infliximab therapy in Crohn's disease. Inflamm Bowel Dis 2011, 17(4), 947-53.

31. Park, Y.; Cheon, J. H.; Park, Y. L.; Ye, B. D.; Kim, Y. S.; Han, D. S.; Kim, J. S.; Hong, S. N.; Kim, Y. H.; Jeon, S. R.; Kim, W. H.; Diseases, I. B. D. S. G. o. t. K. A. f. t. S. o. I., Development of a Novel Predictive Model for the Clinical Course of Crohn's Disease: Results from the CONNECT Study. Inflamm Bowel Dis 2017, 23 (7), 1071-1079.

32. Satsangi, J.; Silverberg, M. S.; Vermeire, S.; Colombel, J. F., The Montreal classification of inflammatory bowel disease: controversies, consensus, and implications. Gut 2006, 55 (6), 749-53.

\section{Figures}


A

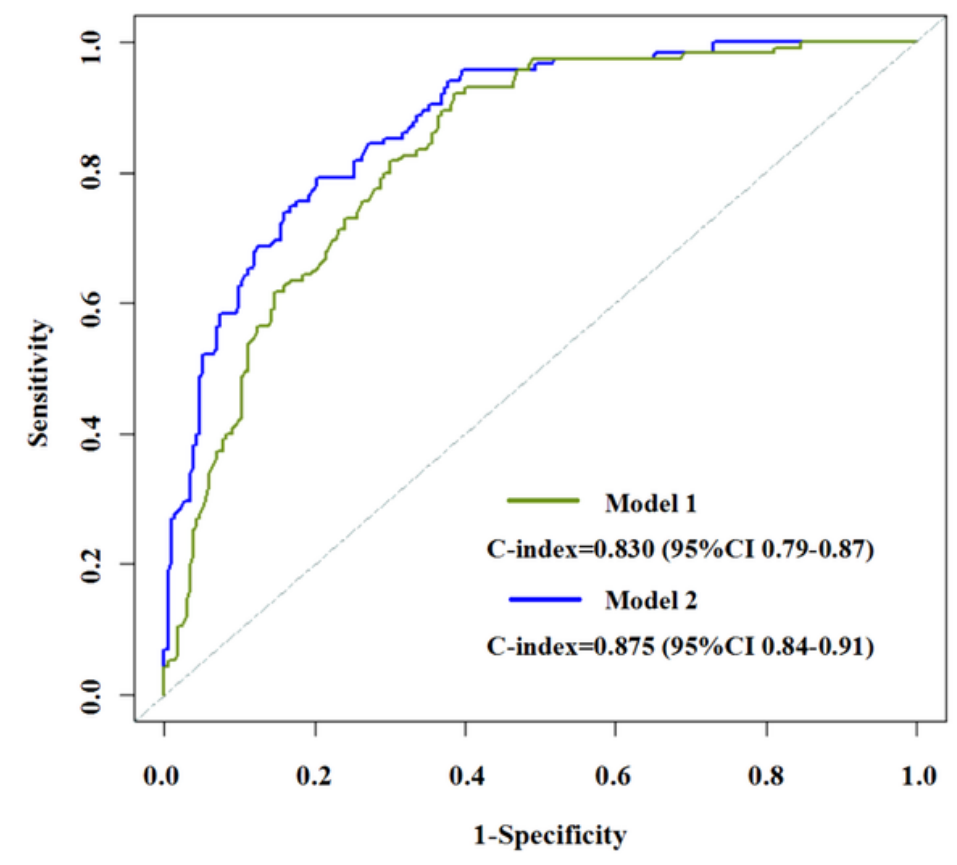

B

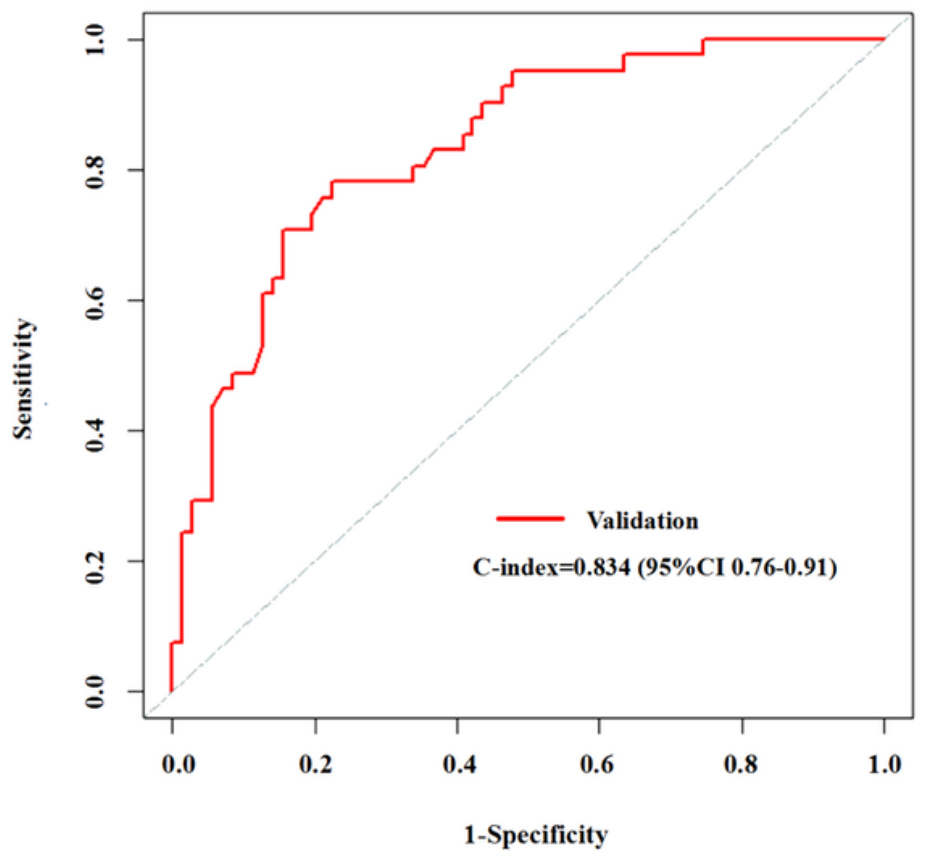

Figure 1

The sensitivity and specificity were 0.626 and 0.807 , respectively (Table.4). Primary model showed a perfect capacity for predicting $\mathrm{MH}$, with a C-index of 0.875 (95\% Cl: 0.84-0.91, $\mathrm{P}<0.001)$ (Fig.1A). After validation, the C-index of the model was 0.834 (95\% Cl: 0.76-0.91, P<0.001) (Fig.1B).

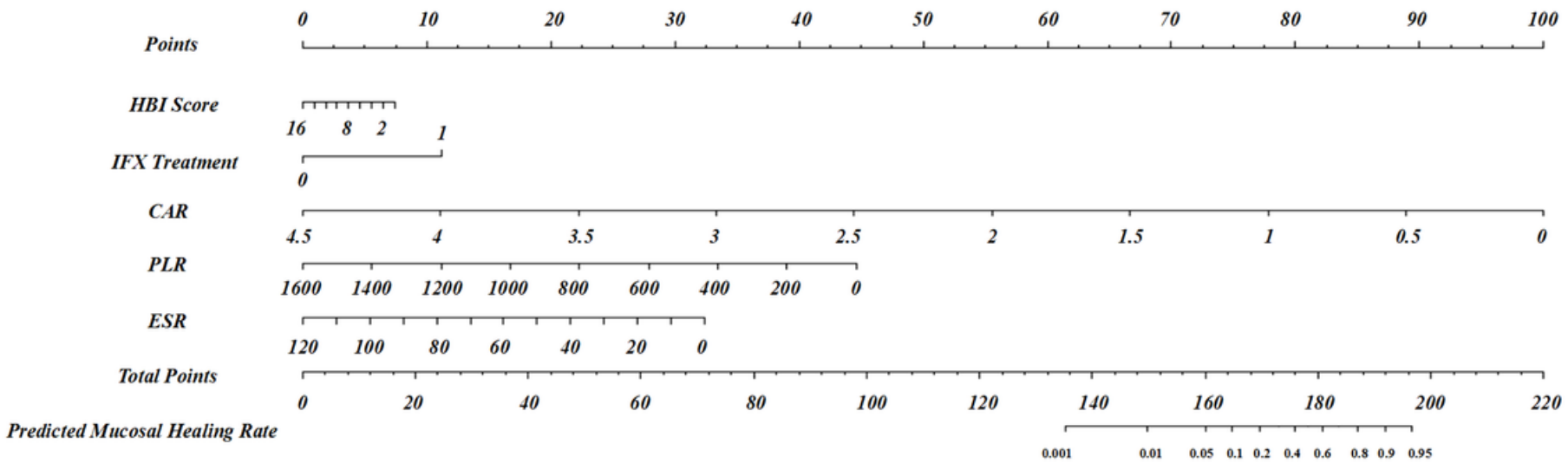

\section{Figure 2}

A nomogram was established based on the variables in the primary model (Fig.2). Model performance was evaluated by discrimination and calibration. This model had a high C-index (0.88) as mentioned above 
A

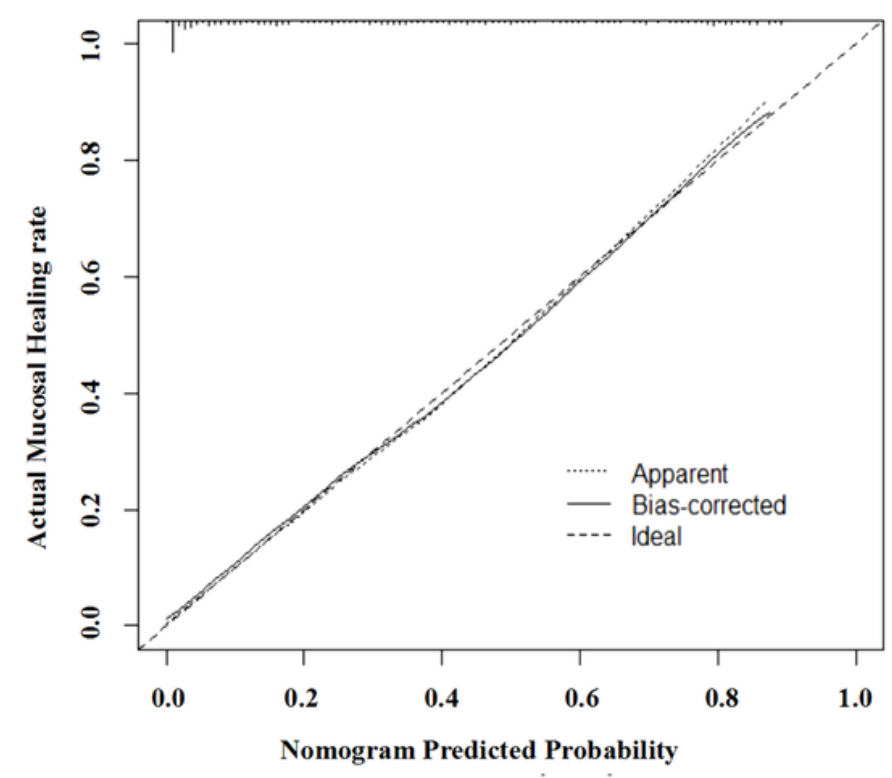

B

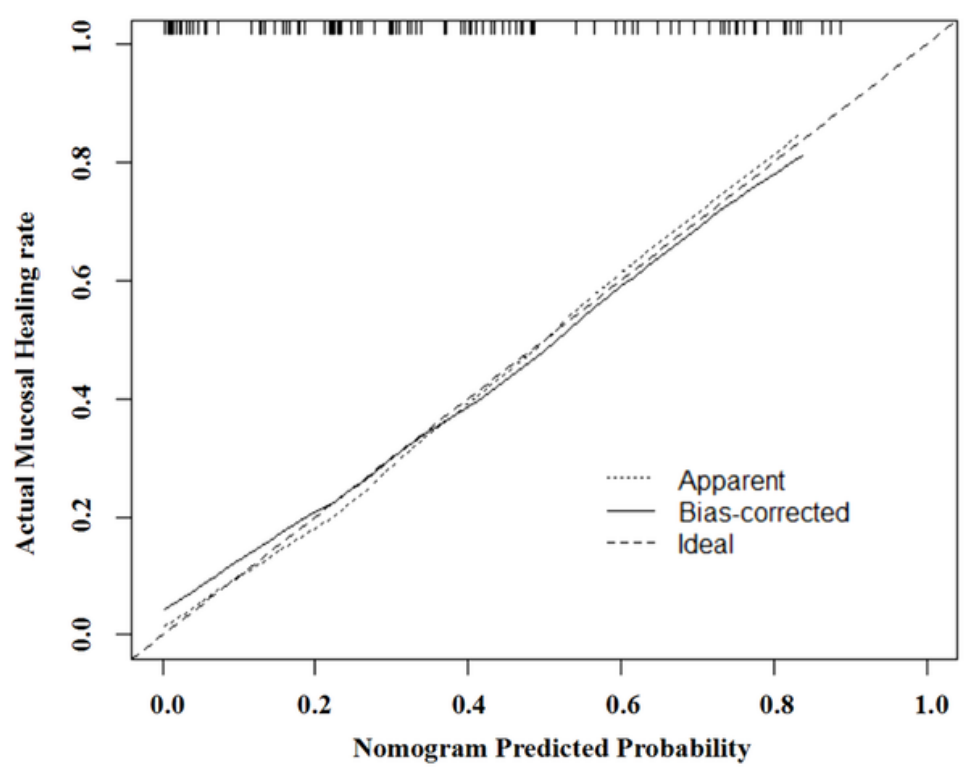

Figure 3

The calibration curve also showed satisfactory performance (figure $3 \mathrm{~A}$ ). The internal validation was performed in $112 \mathrm{CD}$ patients with small bowel involvement. After validation, the $\mathrm{C}$-index of the model was 0.834 (95\% Cl: $0.76-0.91, \mathrm{P}<0.001)$ (Fig.1B). The calibration curve in validation group is shown in Fig.3B. The internal validation also performed good in discrimination and calibration.

\section{Supplementary Files}

This is a list of supplementary files associated with this preprint. Click to download.

- Supplmentarymaterials.docx 